\title{
Neurofibromatose do tipo I na infância: relato de caso clínico
}

\author{
Neurofibromatosis type I in childhood: a clinical case report
}

Neurofibromatosis tipo I en la infancia: reporte de un caso clínico

Leonardo Boundok Martinelli ${ }^{1}$, Rafael Galvão de Dono Tavares ${ }^{1}$, Pedro Henrique Galego Porfirio de Faria Carvalho ${ }^{1}$, Isabella Violante de Oliveira ${ }^{1}$, Mateus de Castro Xavier ${ }^{1}$, Henrique George Naufel ${ }^{1}$, Wagner Alves de Souza Júdice ${ }^{2 *}$.

\section{RESUMO}

Objetivo: Relatar o caso clínico de paciente diagnosticado com Neurofibromatose do tipo I na infância e correlacionar com a literatura a respeito da sintomatologia, diagnóstico e métodos de tratamento. Detalhamentos de Caso: Paciente do sexo masculino, 3 anos e 6 meses, pré-escolar, branco. Ao nascer, foram observadas manchas "café com leite" maiores que $5 \mathrm{~mm}$ de diâmetro. O pediatra então o encaminhou para o dermatologista, que ao suspeitar de neurofibromatose do tipo I, referenciou o paciente para diversas especialidades como a neurologia e oftalmologia. Através de ressonância magnética evidenciou-se a presença de glioma óptico, realizou-se a confirmação das manchas "café com leite" e evidenciaram-se outros sintomas como más formações ósseas. O Diagnóstico foi fechado seguindo os critérios apresentados na literatura médica. Considerações finais: $O$ presente relato demonstra a importância da realização de diagnóstico precoce para manejo correto e acompanhamento adequado do paciente, evitando assim, um impacto negativo ainda maior na qualidade de vida do paciente e de sua família.

Palavras-chave: Neurofibroma, Neurofibromina, Manchas café com leite, Efélide, Nódulos.

\begin{abstract}
Objective: Report the clinical case of a patient diagnosed with Neurofibromatosis type 1 in childhood and correlate with the literature regarding the symptoms, diagnosis and treatment methods. Case Details: Male patient, 3 years and 6 months, preschool, and white. At birth, 'coffee au lait' spots larger than $5 \mathrm{~mm}$ in diameter were observed. The pediatrician then referred him to the dermatologist, who, suspecting type I neurofibromatosis, referred the patient to several specialties such as neurology and ophthalmology. Through magnetic resonance imaging, the presence of optic glioma was evidenced, confirmation of the "coffee au lait" spots was performed, and other symptoms such as bone malformations were evidenced. Diagnosis was closed following the criteria presented in the medical literature. Final considerations: This report demonstrates the importance of carrying out an early diagnosis for correct management and adequate follow-up of the patient, thus avoiding an even greater negative impact on the quality of life of the patient and his family.
\end{abstract}

Key words: Neurofibroma, Neurofibromine, Café-au-lait spots, Ephelid, Nodules.

\section{RESUMEN}

Objetivo: Informar el caso clínico de un paciente diagnosticado de neurofibromatosis tipo I en la infancia y correlacionarlo con la literatura en cuanto a síntomas, diagnóstico y métodos de tratamiento. Detalles del caso: Paciente masculino, 3 años y 6 meses, preescolar, blanco. Al nacer, se observaron manchas de 'café

\footnotetext{
${ }_{1}^{1}$ Faculdade de Medicina da Universidade de Mogi das Cruzes (FM-UMC), Mogi das Cruzes - SP.

${ }^{2}$ Centro Interdisciplinar de Investigação Bioquímica - Universidade de Mogi das Cruzes (CIIB-UMC), Mogi das Cruzes - SP. *E-mail: wagnerjudice@gmail.com
} 
con leche' de más de $5 \mathrm{~mm}$ de diámetro. Posteriormente, el pediatra lo derivó al dermatólogo, quien, sospechando neurofibromatosis tipo I, derivó al paciente a diversas especialidades como neurología y oftalmología. Mediante resonancia magnética se evidenció la presencia de glioma óptico, se realizó la confirmación de las manchas "café con leche" y se evidenciaron otros síntomas como malformaciones óseas. El diagnóstico se cerró siguiendo los criterios presentados en la literatura médica. Consideraciones finales: Este informe demuestra la importancia de realizar un diagnóstico precoz para el correcto manejo y seguimiento adecuado del paciente, evitando así un impacto negativo aún mayor en la calidad de vida del paciente y su familia.

Palabras clave: Neurofibroma, Neurofibromina, Manchas de café con leche, Ephelid, Nódulos.

\section{INTRODUÇÃO}

A Neurofibromatose (NF) é uma desordem genética, do tipo neurocutânea, de caráter autossômico dominante, com expressividade variável e elevada penetrância, podendo acometer todas as raças (AZULAY $\mathrm{RD}, 2013)$. A maioria dos casos dessa doença são decorrentes de herança genética, contudo uma porção considerável de casos provêm de mutações espontâneas, sendo que a última é caracterizada por mutações que ocorrem aleatoriamente em qualquer alelo do DNA (GUTMANN DH, et al., 2017).

Pais com NF apresentam 50\% chances de ter uma criança com NF. As características clínicas do pai doador do gene podem não ser as mesma do filho (NUSSBAUM RL, et al., 2016). NF é classificada em 5 tipos principais: NF-1 ou clássica que é a mais comum compreendendo cerda de $90 \%$ dos casos; NF-2 ou acústica; NF-3 ou segmentar; NF-4 ou MCCL (manchas café com leite) familiar e NF-5 neurofibromatose com fenótipo Noonan (BORTOLUZZI AS, et al., 2017). Estudos epidemiológicos relatam prevalência variando entre $1 / 3000$ e 1/6000, com estimativas de incidência em nascidos vivos entre 1/2558 e 1/3333. Recentemente verifvicou-se que NF1 é um transtorno muito mais comum do que se pensava, com incidência de nascimento de 1:2000 e prevalência de 1/4000 (UUSITALO E, et al., 2015; KALLIONPAA RA, et al., 2018; BERGQVIST C, et al., 2020)

O gene NF1 responsável pela doença está localizado no cromossomo 17 no locus $17 q 11.2$ e codifica a proteína neurofibromina (POSWAL P, et al., 2020). As manifestações clínicas da doença são sistêmica e progressiva afetando principalmente pele, sistema nervoso, ossos e os olhos, dentre outros orgãos (ALVES JÚNIOR SF, et al. 2019).

O gene NF-1 possui 59 éxons sendo responsáveis pela produção da neurofibromina (BORTOLUZZI AS, et al., 2017). A proteína é expressa em quase todos os tecidos humano, estando mais presente no cérebro, medula espinhal, sistema nervoso periférico e nervos. A neurofibromina atua como um gene supressor de tumor por regulação negativa do produto do gene RAS. Sua mutação resulta na proliferação de vários neurofibromas e outros tumores (THEOS A e KORF BR, 2017). A neurofibromina estimula a atividade RasGTPase para convertê-la de uma forma ligada a GTP ativa em sua forma ligada a GDP inativa por meio de seu domínio relacionado à proteína ativadora de GTPase (GRD) (TAO J, et al., 2020).

Dentre as diversas expressões clínicas, algumas características são básicas para identificação dessa doença. As manchas café com leite surgem em $90 \%$ dos casos como sintoma pioneiro, a segunda principal manifestação, a surgir na criança, são as efélipes (máculas pigmentadas) (FARSCHTSCHI S, et al., 2020).

Para o diagnostico completo dessa desordem genética é necessário seguir alguns critérios clínicos específicos, e averiguar a presença de dois ou mais destes critérios, sendo eles: dois ou mais neurofibromas de qualquer tipo, pelo menos um neurofibroma plexiforme, efélides nas regiões axilares e/ou inguinais, um glioma do nervo óptico, dois ou mais nódulos de Lisch, uma lesão óssea distinta, como displasia esfenoidal ou pseudartrose tibial, seis ou mais manchas café com leite iguais ou maiores que $5 \mathrm{~mm}$ de diâmetro em indivíduos abaixo de 6 anos e maiores que $15 \mathrm{~mm}$ de diâmetro em indivíduos acima de 6 anos, um parente de primeiro grau com a mesma doença e com o mesmo tipo (NF-1) (BORTOLUZZI AS, et al., 2017; MIRAGLIA E, et al., 2020). 
Associado a estes critérios, é importante destacar alguns parâmetros físicos, tanto relacionados ao exame físico do paciente, quanto ao exame intelectual, se destacam: qualidade de aprendizagem e progresso estudantil, vivência social com outras crianças, alterações visuais, comprimento do perímetro cefálico, altura, peso e desenvolvimento musculoesquelético (FARSCHTSCHI S, et al., 2020). Indivíduos que possuem um diagnóstico familiar de NF-1 podem ser diagnosticadas clinicamente no primeiro ano de vida uma vez que é necessária apenas uma característica da doença presente. Contudo, indivíduos que não possuem uma história familiar prévia, terão atingidos os critérios mínimos diagnósticos até oito anos de idade (NUSSBAUM $\mathrm{RL}$, et al., 2016).

O selumetinib é o primeiro medicamento aprovado para tratamento de pacientes pediátricos com 2 anos ou mais de idade com NF-1. O medicamento é um inibidor de quinase que ajuda a interromper o crescimento do tumor neurofibroma ao bloquear uma enzima chave. É indicado especificamente para pacientes com neurofibromas plexiformes sintomáticos e inoperáveis, que envolvem as bainhas nervosas e podem crescer em qualquer parte do corpo, incluindo ao redor da coluna e em áreas onde podem afetar órgãos internos (VOELKER R, 2020).

Algumas características do NF-1 podem estar presentes no nascimento, mas a maioria das manifestações surge com a idade, necessitando de monitoramento periódico para atender às necessidades contínuas de saúde e desenvolvimento e minimizar o risco de complicações médicas graves (MILLER DT, et al., 2019). Diagnóstico de NF-1 é baseado nos critérios do National Institutes of Health (NIH). As características clínicas importantes de seu diagnóstico incluem o tamanho e o número de manchas café com leite e sardas, neurofibromas cutâneos, nódulos de Lisch nos olhos e história de família. No entanto, alguns pacientes não atendem aos critérios do $\mathrm{NIH}$ para diagnóstico; por exemplo, alguns pacientes pediátricos apresentam apenas algumas manchas café com leite de tamanho variável. Com a idade de 1 ano, apenas cerca de $50 \%$ dos bebês sem histórico familiar de NF-1 atenderão a esses critérios (KANG E, et al., 2021).

A Clínica de Neurofibromatose mantém estreita ligação com outras especialidades devido aos encaminhamentos frequentes devido ao curso natural da doença (Oftalmologia Pediátrica, Neuro-Oncologia Pediátrica, Neurocirurgia, Genética Médica, Unidade de Desenvolvimento Infantil e Ortopedia) (NISHIDA Y, et al., 2021).

Nesse sentido, o objetivo desse estudo foi realizar um relato de caso clínico de paciente diagnosticado com Neurofibromatose do tipo I na infância e correlacionar com a literatura a respeito da sintomatologia, diagnóstico e métodos de tratamento.

\section{DETALHAMENTO DO CASO}

O relato de caso apresentado nesse estudo foi avaliado pelo Comitê de Ética em Pesquisa da Universidade de Mogi das Cruzes sob protocolo CAAE número 35335220.5.0000.5497 e parecer de aprovação número 4.245.614.

Paciente do sexo masculino, 3 anos e 6 meses, pré-escolar, branco, primeiro filho de casal não consanguíneo. Ao nascer, foram observadas manchas "café com leite" maiores que $5 \mathrm{~mm}$ de diâmetro. Nasceu de cesárea, 39 semanas, pesando 3875 gramas e comprimento de $52 \mathrm{~cm}$ com perímetro cefálico de $35 \mathrm{~cm}$ e APGAR de 8/9, além disso, não fez transfusões sanguíneas, antibioticoterapia ou fototerapia. Paciente realizou aleitamento materno exclusivo e foi corretamente vacinado.

A progenitora do paciente teve somente uma gestação durante a vida, sendo esta uma cesárea. Realizou 6 consultas pré-natal em ambulatório especializado, relatando que não consumiu álcool, drogas ilícitas ou abortivos, além disso negou tabagismo durante o período gestacional. Não teve intercorrências durante a gestação e não necessitou utilizar nenhuma medicação, assim como não desenvolveu nenhuma doença. Como recomendado na literatura, a mãe do paciente utilizou ácido fólico e realizou o ultrassom durante o período de gravidez. Ademais, durante o parto, afirma que foi realizada tentativa de parto normal, mas após 12 horas de bolsa rompida, decidiram pela cesárea e para realização desse procedimento foi utilizado soro de ocitocina sintética. 
Na primeira consulta de puericultura realizada com aproximadamente 1 mês de vida, a mãe relatou observar uma massa na região inguinal cuja suspeita foi de hérnia inguinal, além de sobreposição dos dedos dos pés. Foi destacado ainda na consulta, manchas café com leite em diversas regiões, já notadas anteriormente no momento pós-parto. Foi solicitado acompanhamento com o dermatologista para avaliação inicial das manchas.

Posteriormente, na avaliação com o dermatologista, já com 8 meses, foi dada a hipótese inicial de neurofibromatose do tipo I já que o paciente apresentava mais de 6 manchas hipercrômicas maiores de $5 \mathrm{~mm}$ de diâmetro, sendo menor de $6 \mathrm{~mm}$. Dessa forma, foi requisitada avaliação com o oftalmologista e neurologista pediátrico, especialidades típicas de um paciente com neurofibromatose, para procurar outros sinais típicos desse quadro. É importante destacar que as manchas não tiveram evolução com o passar dos meses (Figura 1).

Figura 1 - Sinais clínicos de neurofibromatose do tipo 1 (manchas café com leite).

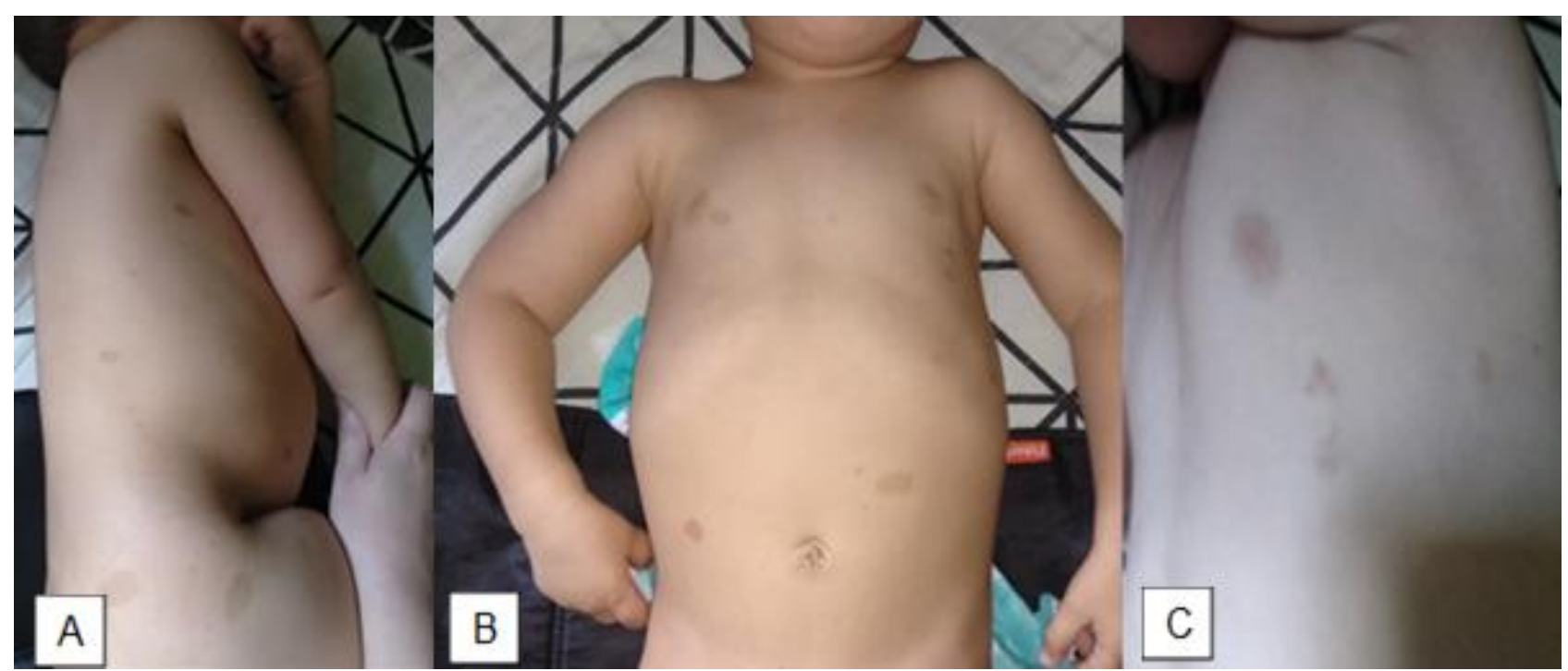

Legenda: A: vista lateral direita; B: vista anterior do tórax e abdome; C: vista lateral esquerda.

Fonte: Martinelli LB, et al., 2021.

Nas diversas consultas com o neurologista, foi solicitada a realização de uma ressonância magnética que foi postergada por diversas vezes devido a motivos pessoais e familiares. Ainda foi relatado atraso no desenvolvimento cognitivo. Finalmente, em julho de 2020, aos 2 anos e 5 meses, paciente realizou a ressonância magnética, cujo laudo reportava a formação expansiva e infiltrativa que afetava o tronco encefálico e se espalhava para os hemisférios cerebelares através dos pedúnculos cerebelares médios à esquerda, sem destaque notável ao contraste paramagnético medindo 2,7 x 1,3 cm, caracterizada por hipersinal em T1 e T2, condicionando moderado efeito compressivo com diminuição das dimensões do quarto ventrículo.

A lesão apresentava mensuração limitada devido a seu aspecto infiltrativo, com tamanho de 4,9 x 3,9 cm, nos maiores eixos axiais no nível dos pedúnculos cerebrais médios. Os achados do paciente não eram exatos, porém pode-se considerar entre as hipóteses mais prováveis astrocitoma do tronco encefálico. No contexto clínico do paciente, foi considerada também a possibilidade de glioma do nervo óptico. Não houve restrição à difusão, não houve hemorragia intracraniana, os flow-voids das principais artérias intracranianas estavam preservados (Figura 2).

Em consultas regulares com o oftalmologista, o paciente sempre apresentou exame oftalmológico dentro da normalidade para idade, incluso postura visual, biomicroscopia, motilidade ocular e mapeamento de retina (Figura 2). 
Figura 2 - Ressonância magnética evidenciando formação expansiva e infiltrativa, tendo como possibilidade um glioma do nervo óptico.

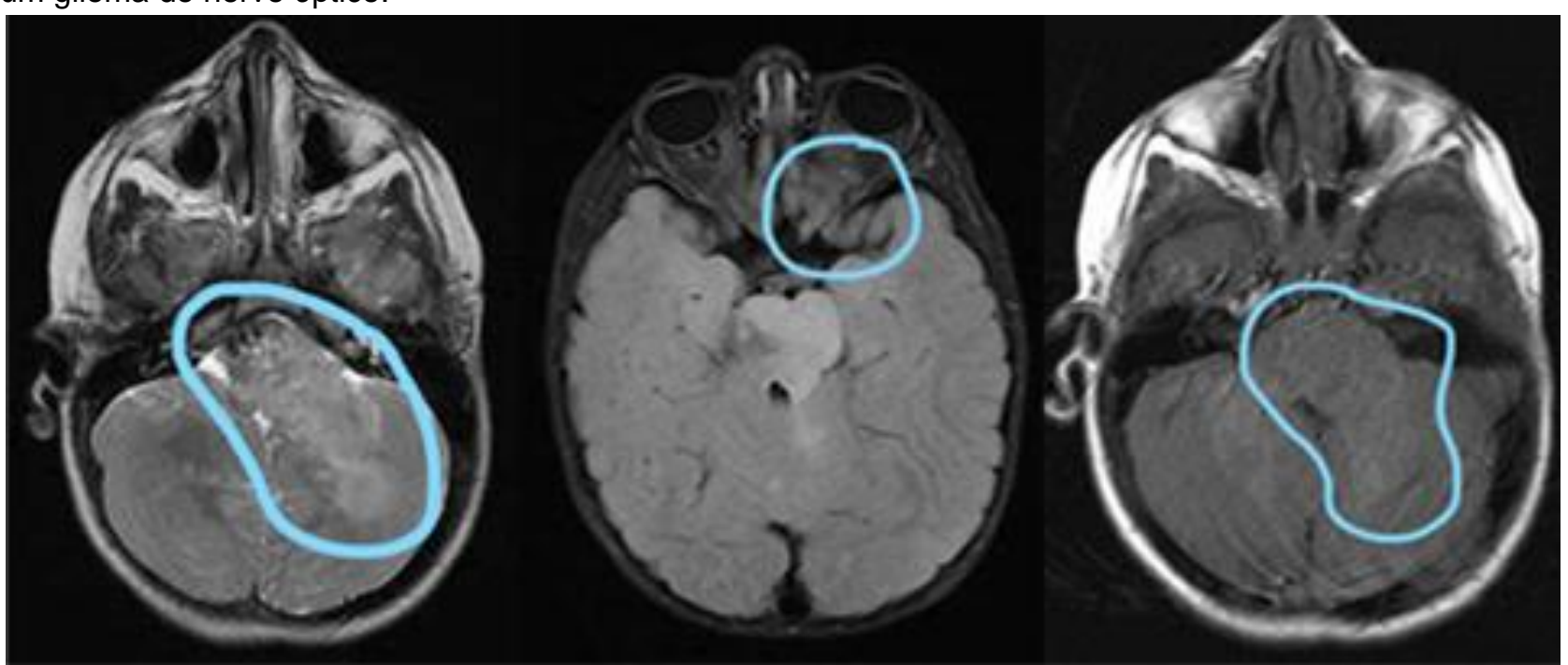

Fonte: Martinelli LB, et al., 2021.

Por fim, em consultas com o ortopedista, foi feita a radiografia já que é comum alterações ósseas nesse quadro e verificou-se dedo em gatilho no primeiro quirodáctilo da mão direita. Além disso, foi solicitado para o paciente uma avaliação futura com o fonoaudiólogo (Figura 3).

Figura 3 - Radiografia da mão direita evidenciando dedo em gatilho no primeiro quirodáctilo.

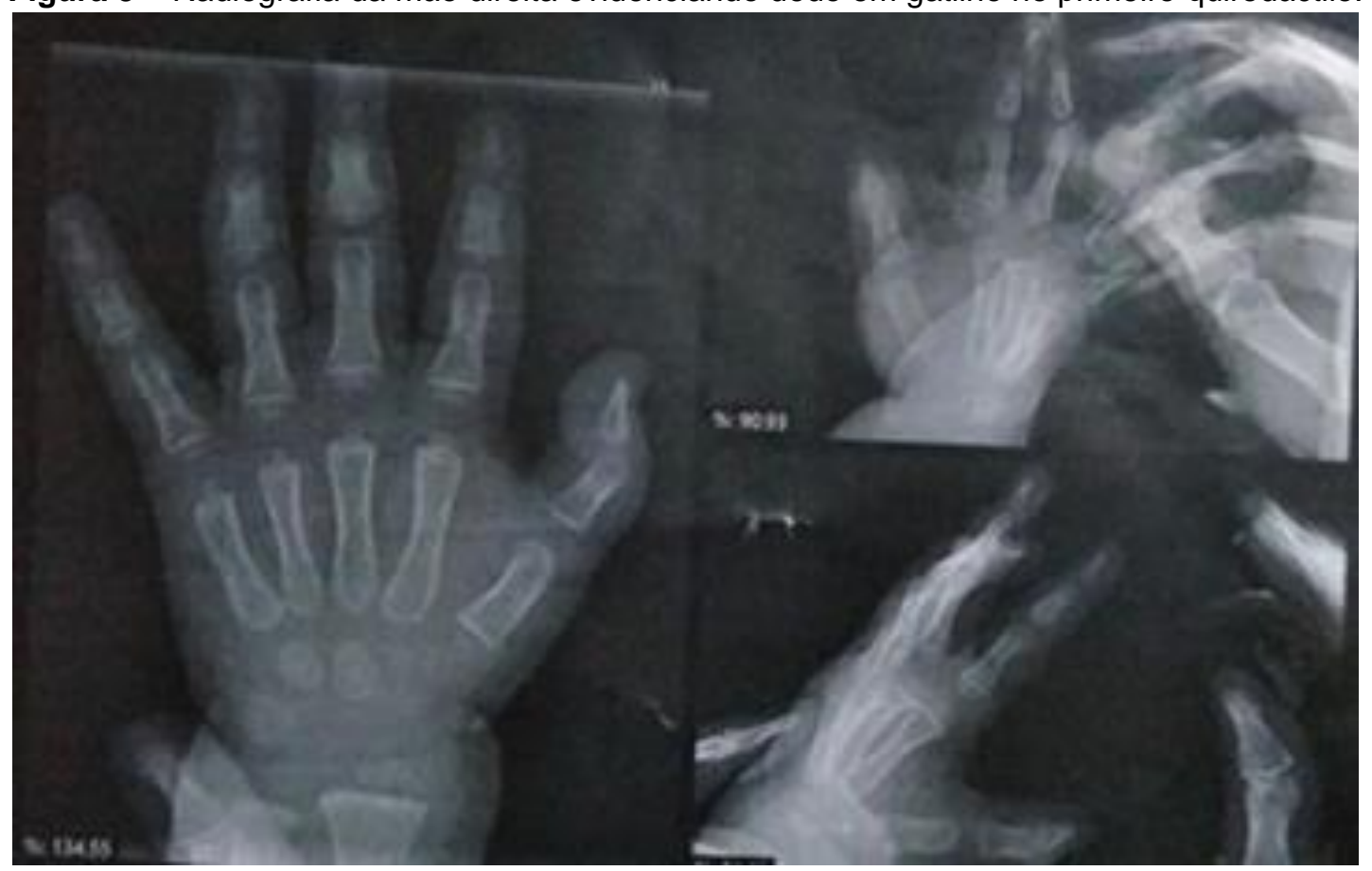

Fonte: Martinelli LB, et al., 2021.

O paciente está sendo acompanhado pelos ambulatórios de neurologia para possibilidades de retirada de neurofibromas de crescimento rápido; dermatologia para avaliação das manchas café com leite pela e orientações para hidratação; ortopedia com consultas rotineiras monitorando surgimento de más formações ósseas e ou complicações daquelas já existentes. Verificou-se melhoras em sua qualidade de vida e houve reinserção social na escola, evidenciando mais uma vez a importância do diagnóstico precoce para a 
implementação de medidas de redução de danos. Além disso, mantém acompanhamento com fonoaudióloga, obtendo resultados favoráveis e evoluções significativas para seu caso.

\section{DISCUSSÃO}

Por se tratar de uma alteração genética com a possibilidade de diagnóstico clínico final que pode se estender até oito anos de idade. Nesse caso, comparando-se os critérios clínicos específicos com os sinais que o paciente apresentava os mais indicativos para o diagnóstico final de neurofibromatose do tipo I foram: presença de seis ou mais manchas café com leite maiores que $5 \mathrm{~mm}$ de diâmetro, presença indicativa de glioma óptico, alteração do comprimento do perímetro cefálico que estava levemente aumentado e por fim, atraso no desenvolvimento cognitivo relatado pela responsável durante as consultas (BORTOLUZZI AS, et al., 2017).

A neurofibromatose do tipo 1, além de apresentar danos fisiológicos ao organismo, afeta também drasticamente as relações sociais do paciente, já que existem alterações dermatológicas visíveis e a diminuição da velocidade do desenvolvimento cognitivo, que pode impactar diretamente não apenas na vida escolar da criança, mas também em seu futuro profissional (DOSER K, et al., 2019). Nesse caso, a responsável relatou dificuldade na relação de seu filho com as funcionárias da creche, principalmente nos momentos de refeição, já que existe uma grande comparação por parte delas entre o desenvolvimento do paciente em questão e as outras crianças.

Dessa forma, se torna necessário e benéfico o diagnóstico precoce da neurofibromatose, para se iniciar um acompanhamento, principalmente dermatológico, oftalmológico, neurológico e ortopédico (NISHIDA Y, et al., 2021). Assim, o pediatra conseguirá observar as evoluções específicas de cada paciente. Ademais, a orientação psicológica aos pais e à criança é essencial desde o momento do diagnóstico. Com todos esses atendimentos, as possíveis complicações da neurofibromatose do tipo 1 se reduzem, melhorando a qualidade de vida não só do doente, mas também da família (SANAGOO A, et al., 2019).

O paciente já frequentava constantemente o dermatologista, oftalmologista e neurologista, entretanto, com a verificação do surgimento do glioma de nervo óptico, por ressonância magnética em julho de 2020, isso especificou os indícios de neurofibromatose do tipo 1, pois, $15 \%$ dos pacientes com neurofibromatose apresentam esse achado clínico, entretanto apenas $5 \%$ desses pacientes desenvolvem sintomas. Embora, 0 paciente em questão tem o indicativo de glioma óptico, se mantém assintomático. Em relação ao glioma, majoritariamente são do tipo astrocitomas pilocíticos do nervo óptico, entretanto podem se apresentar também em quiasma e tratos ópticos. Normalmente, os gliomas ópticos evoluem muito lentamente, são estáveis e podem regredir espontaneamente (LOBBOUS M, et al., 2020).

O astrocitoma, tipo de glioma, como já referido anteriormente, é caracterizado por se apresentar difusamente com alto ou baixo grau. No presente caso, ocorreram ainda focos de hipersinal em T1 e T2, que são anormalidades não neoplásicas no parênquima cerebral. São benignas e percebida em aproximadamente 2/3 dos pacientes, sendo comum no tronco encefálico, exatamente onde foi notado no paciente. Ademais, não foi observado restrição à difusão, nem hemorragia intracraniana, e os flow-voids das principais artérias cranianas estavam preservados, o que facilitou o prognóstico (D'ANGELO F, et al., 2019).

Desde a descoberta da formação expansiva infiltrativa na ressonância magnética, o acompanhamento médico está sendo ainda mais constante, com avaliações periódicas para identificação de surgimento de neurofibromas, detalhamento do neurodesenvolvimento, atenção ao desenvolvimento ósseo e possível surgimento de anormalidades de membros (BORTOLUZZI AS, et al., 2017).

Uma particularidade encontrada nesse paciente foi a presença de dedo em gatilho, que é caracterizado como um bloqueio dos tendões flexores dos dedos, e essa doença também é conhecida como tenossinovite estenosante, e o dedo mais comumente afetado é o quirodáctilo (MEHLMANN FM, et al., 2019), fato este que ocorreu com o paciente desse caso clínico. Além disso, esse paciente apresentou um quadro indolor com curvatura em flexão das articulações interfalangianas. O diagnóstico foi essencialmente clínico, sendo que a necessidade de radiografia serviu somente para descartar problemas osteoarticulares. 
A base do manejo da NF-1 é um cuidado longitudinal centrado no paciente com monitoramento específico da idade das manifestações clínicas, visando o reconhecimento precoce e o tratamento sintomático das complicações à medida que ocorrem. O engajamento ativo e uma parceria ativa entre vários prestadores de cuidados de saúde, grupos leigos interessados e especialistas em pacientes é a pedra angular da gestão desta doença rara (BERGQVIST C, et al., 2020).

O monitoramento vitalício é recomendado assim que houver suspeita do diagnóstico de NF-1. A avaliação clínica por um especialista em NF-1 deve ocorrer anualmente para crianças e adultos com fenótipo de alto risco. Caso contrário, os pacientes NF-1 sem o fenótipo de alto risco ou complicações devem visitar o especialista em NF-1 a cada dois a 3 anos, com o restante das visitas ocorrendo anualmente com um médico de atenção primária, dermatologista ou pediatra (CENTRE DE REFERENCE LABELLISE NEUROFIBROMATOSES, 2016).

Sendo uma doença rara, os portadores de NF-1 necessitam de um diagnóstico precoce e acompanhamento constante de multi especialidades médicas. Verificou-se que o paciente apresentava manchas de café com leite, atraso no desenvolvimento cognitivo, glioma óptico, alteração do comprimento do perímetro cefálico, dedo em gatilho no primeiro quirodáctilo da mão direita. O conjunto de informações do quadro clínico permitiu o diagnóstico de NF-1, e o paciente continua em acompanhamento em diversas especialidades.

\section{REFERÊNCIAS}

1. ALVES JÚNIOR SF, et al. Neurofibromatosis type 1: State-of-the-art review with emphasis on pulmonary involvement. Respir Med, 2019; 149: 9-15.

2. AZULAY RD. Dermatologia. 6. ed. Rio de Janeiro: Guanabara Koogan, 2013; 1156p.

3. BERGQVIST C, et al. Neurofibromatosis 1 French national guidelines based on an extensive literature review since 1966. Orphanet J Rare Dis, 2020; 15(1): 37.

4. BORTOLUZZI AS, et al. Neurofibromatose tipo 1 - impactos na infância e adolescência. Faz Ciência, 2017; 19(29): 81-104.

5. CENTRE DE REFERENCE LABELLISE NEUROFIBROMATOSES. Protocole National de Diagnostic et de Soins. $\begin{array}{llll}\text { Neurofibromatose } & 1 . & 2016 . & \text { Disponível }\end{array}$ http://www.orpha.net/data/patho/PNDS/Neurofibromatose_type_1_FR_fr_PNDS.pdf.

6. D'ANGELO F, et al. The molecular landscape of glioma in patients with Neurofibromatosis 1. Nat Med. 2019; 25(1):176-87.

7. DOSER K, et al. Educational delay and attainment in persons with neurofibromatosis 1 in Denmark. Eur. J. Hum. Genet, 2019; 27: 857-68.

8. FARSCHTSCHI S, et al. The Neurofibromatoses. Dtsch Arztebl Int, 2020; 117(20): 354-60.

9. GUTMANN DH, et al. Neurofibromatosis type 1. Nature Reviews Disease Primers, 2017; 3: 17004.

10. KANG E, et al. Neurofibromatosis type I: points to be considered by general pediatricians. Clin Exp Pediatr, 2021, 64(4): 149-56.

11. KALLIONPAA RA, et al. Prevalence of neurofibromatosis type 1 in the Finnish population. Genitourin Med, 2018; 20(9): 1082-6.

12. LOBBOUS M, et al. An Update on Neurofibromatosis Type 1-Associated Gliomas. Cancers (Basel), $2020 ; 12(1): 114$.

13. MILLER DT, et al. Health Supervision for Children with Neurofibromatosis Type 1. Pediatrics, 2019 ; e20190660.

14. MIRAGLIA E, et al. Cutaneous manifestations in neurofibromatosis type 1. Clin Ter. 2020; 171(5): e371-e377.

15. NISHIDA Y, et al. Establishment of in-hospital clinical network for patients with neurofibromatosis type 1 in Nagoya University Hospital. Sci Rep, 2021; 11: 11933.

16. NUSSBAUM RL. Thompson \& Thompson Genética Médica. 8. ed. Rio de Janeiro: Elsevier, 2016; 560p.

17. POSWAL $P$, et al. Plexiform neurofibroma with neurofibromatosis type I/ von Recklinghausen's disease: A rare case report. Annals of Medicine and Surgery, 2020; 57: 346-50.

18. SANAGOO A, et al. Evaluation of QoL in neurofibromatosis patients: a systematic review and meta-analysis study. BMC Neurol, 2019; 19: 123.

19. TAO J, et al. Advancement in research and therapy of NF1 mutant malignant tumors. Cancer Cell International, 2020; 20: 492.

20. THEOS A, KORF BR. Reference Module in Neuroscience and Biobehavioral Psychology II - Neurofibromatosis Type 1. Elsevier, 2017.

21. UUSITALO E, et al. Incidence and mortality of neurofibromatosis: a total population study in Finland. J Invest Dermatol, 2015; 135(3): 904-6.

22. VOELKER R. A New Treatment for Children with Neurofibromatosis Type 1. J Am Med Assoc-Jama, 2020; 323(19): 1887. 